\title{
Stellar Occultation by Comet 67P/Churyumov-Gerasimenko Observed with Rosetta's Alice Far-ultraviolet Spectrograph
}

\author{
Brian A. Keeney ${ }^{1}$ (D) S. Alan Stern ${ }^{1}$, Paul D. Feldman ${ }^{2}$ (1) Michael F. A'Hearn ${ }^{3,10}$, Jean-Loup Bertaux ${ }^{4}$, Lori M. Feaga ${ }^{3}$, \\ Matthew M. Knight ${ }^{3}$ (D), Richard A. Medina ${ }^{1}$, John Noonan ${ }^{5}$ (D), Joel Wm. Parker ${ }^{1}$, Jon P. Pineau ${ }^{6}$, Rebecca N. Schindhelm ${ }^{1,7}$, \\ Andrew J. Steffl ${ }^{1}$ (D), M. Versteeg ${ }^{8}$, Ronald J. Vervack, Jr. ${ }^{9}$ (D), and Harold A. Weaver ${ }^{9}$ (D) \\ ${ }^{1}$ Southwest Research Institute, Department of Space Studies, Suite 300, 1050 Walnut Street, Boulder, CO 80302, USA; bkeeney@gmail.com \\ ${ }^{2}$ Department of Physics and Astronomy, Johns Hopkins University, 3400 N. Charles Street, Baltimore, MD 21218, USA \\ ${ }^{3}$ Department of Astronomy, University of Maryland, College Park, MD 20742, USA \\ ${ }^{4}$ LATMOS, CNRS/UVSQ/IPSL, 11 Boulevard d'Alembert, F-78280 Guyancourt, France \\ ${ }^{5}$ Lunar and Planetary Laboratory, University of Arizona, 1629 E. University Boulevard, Tucson, AZ 85721, USA \\ ${ }^{6}$ Stellar Solutions, Inc., 250 Cambridge Ave., Suite 204, Palo Alto, CA 94306, USA \\ ${ }^{7}$ Ball Aerospace and Technology Corp., 1600 Commerce Street, Boulder, CO 80301, USA \\ ${ }^{8}$ Southwest Research Institute, 6220 Culebra Road, San Antonio, TX 78238, USA \\ ${ }^{9}$ Space Exploration Sector, Johns Hopkins University Applied Physics Laboratory, 11100 Johns Hopkins Road, Laurel, MD 20723, USA \\ Received 2018 November 8; revised 2019 March 15; accepted 2019 March 15; published 2019 April 12
}

\begin{abstract}
Following our previous detection of ubiquitous $\mathrm{H}_{2} \mathrm{O}$ and $\mathrm{O}_{2}$ absorption against the far-ultraviolet continuum of stars located near the nucleus of Comet 67P/Churyumov-Gerasimenko, we present a serendipitously observed stellar occultation that occurred on 2015 September 13, approximately one month after the comet's perihelion passage. The occultation appears in two consecutive 10-minute spectral images obtained by Alice, Rosetta's ultraviolet $\left(700-2100 \AA\right.$ ) spectrograph, both of which show $\mathrm{H}_{2} \mathrm{O}$ absorption with column density $>10^{17.5} \mathrm{~cm}^{-2}$ and significant $\mathrm{O}_{2}$ absorption $\left(\mathrm{O}_{2} / \mathrm{H}_{2} \mathrm{O} \approx 5 \%-10 \%\right)$. Because the projected distance from the star to the nucleus changes between exposures, our ability to study the $\mathrm{H}_{2} \mathrm{O}$ column density profile near the nucleus (impact parameters $<1 \mathrm{~km}$ ) is unmatched by our previous observations. We find that the $\mathrm{H}_{2} \mathrm{O}$ and $\mathrm{O}_{2}$ column densities decrease with increasing impact parameter, in accordance with expectations, but the $\mathrm{O}_{2}$ column decreases $\sim 3$ times more quickly than $\mathrm{H}_{2} \mathrm{O}$. When combined with previously published results from stellar appulses, we conclude that the $\mathrm{O}_{2}$ and $\mathrm{H}_{2} \mathrm{O}$ column densities are highly correlated, and $\mathrm{O}_{2} / \mathrm{H}_{2} \mathrm{O}$ decreases with the increasing $\mathrm{H}_{2} \mathrm{O}$ column.
\end{abstract}

Key words: comets: individual (67P) - ultraviolet: planetary systems

\section{Introduction}

The double-focusing mass spectrometer (DFMS) of the Rosetta Orbiter Spectrometer for Ion and Neutral Analysis (ROSINA; Balsiger et al. 2007) has found that $\mathrm{O}_{2}$ is the fourth most abundant parent species in the coma of Comet 67P/ Churyumov-Gerasimenko (67P/C-G), behind only $\mathrm{H}_{2} \mathrm{O}, \mathrm{CO}_{2}$, and CO (Le Roy et al. 2015; Fougere et al. 2016). The ubiquitous, abundant presence of $\mathrm{O}_{2}$ was surprising since it had never been detected in a comet before (Bieler et al. 2015).

Feldman et al. (2016) confirmed the presence of substantial $\mathrm{O}_{2}$ in the coma of $67 \mathrm{P} / \mathrm{C}-\mathrm{G}$ during gaseous outbursts with Alice, Rosetta's ultraviolet spectrograph (Stern et al. 2007). Later, Keeney et al. (2017) directly detected $\mathrm{O}_{2}$ absorption in the Alice data using stellar sight lines temporarily projected near the nucleus (stellar appulses). These sight lines were observed over a wide range of heliocentric distances $(1.2-2.3 \mathrm{au})$ and impact parameters $(4-20 \mathrm{~km})$, yielding $\log N_{\mathrm{H}_{2} \mathrm{O}}=15.2-17.1$ (all column densities, $N$, herein are quoted in units of $\mathrm{cm}^{-2}$ ) and a median value of $N_{\mathrm{O}_{2}} / N_{\mathrm{H}_{2} \mathrm{O}}=25 \%$.

Several Rosetta instruments can directly detect $\mathrm{H}_{2} \mathrm{O}$ in the coma of 67P/C-G: Alice, ROSINA, the Visible and Infrared Thermal Imaging Spectrometer (VIRTIS; Coradini et al. 2007), and the Microwave Instrument for the Rosetta Orbiter (MIRO; Gulkis et al. 2007). All but ROSINA are remote-sensing instruments that measure or infer column densities along a line

\footnotetext{
${ }^{10}$ Deceased.
}

of sight, and $N_{\mathrm{H}_{2} \mathrm{O}}$ measured by Alice (Keeney et al. 2017) agrees with VIRTIS values near perihelion (Bockelée-Morvan et al. 2016). However, only Alice and ROSINA can directly detect $\mathrm{O}_{2}$, and the relative abundance of $\mathrm{O}_{2} / \mathrm{H}_{2} \mathrm{O}$ in the Alice data was nearly an order of magnitude larger than the average ROSINA value $\left(n_{\mathrm{O}_{2}} / n_{\mathrm{H}_{2} \mathrm{O}}=3.85 \pm 0.85 \%\right.$, where $n$ is number density measured at the spacecraft position; Bieler et al. 2015). The consistency in $N_{\mathrm{H}_{2} \mathrm{O}}$ between Alice and VIRTIS suggests that the methodology of Keeney et al. (2017) is trustworthy, but the discrepancy in $\mathrm{O}_{2} / \mathrm{H}_{2} \mathrm{O}$ remains puzzling.

Here we present a stellar occultation by $67 \mathrm{P} / \mathrm{C}-\mathrm{G}$ observed with Alice. We describe our observations in Section 2 and our analysis procedure in Section 3. Section 4 compares the $\mathrm{H}_{2} \mathrm{O}$ and $\mathrm{O}_{2}$ column densities for our stellar occultation with those of Keeney et al. (2017) and the ROSINA measurements (Bieler et al. 2015; Hansen et al. 2016) and Section 5 summarizes our main findings.

\section{Observations}

The A0 IV star HD 4150 was occulted by 67P/C-G on 2015 September 13, approximately one month after the comet's perihelion passage. The occultation was serendipitously observed by Alice during the course of normal operations. During this phase of the mission, Alice was integrating nearly continuously to catalog emissions from the near-nucleus coma (Pineau et al. 2019). 


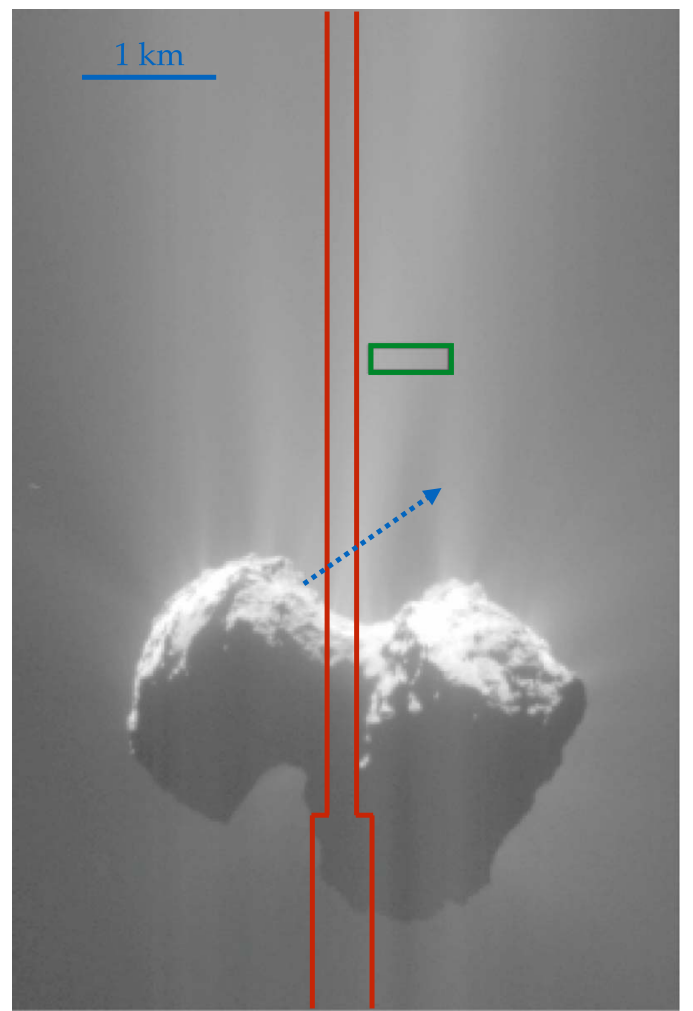

Figure 1. Subsection of a NAVCAM image taken while HD 4150 was occulted by $67 \mathrm{P} / \mathrm{C}-\mathrm{G}, \sim 20$ minutes before the star entered the Alice slit (red). The VIRTIS-H aperture is shown in green, and the approximate path of HD 4150 through the Alice slit is shown by a dashed blue arrow. The image is oriented such that the Sun is toward the top, and the field of view is $\sim 1^{\circ} \times 1.5$ $\left(\sim 5 \times 7.5 \mathrm{~km}^{2}\right)$.

Figure 1 shows a $\sim 5 \times 7.5 \mathrm{~km}^{2}$ subsection of a Rosetta navigation camera (NAVCAM) image taken at 13:36:02 UT, while HD 4150 was occulted by $67 \mathrm{P} / \mathrm{C}-\mathrm{G}$ and $\sim 20$ minutes before it entered the Alice slit. The Alice slit is $5^{\circ} .5$ long and has a dog-bone shape that is twice as wide at the top and bottom as in the center (Stern et al. 2007); its position with respect to the nucleus is shown in red in Figure 1, and the transition between the wide-bottom and narrow-center regions of the slit is evident. Figure 1 also shows the approximate path of HD 4150 as it emerges from behind the small lobe of $67 \mathrm{P} /$ $\mathrm{C}-\mathrm{G}$ and crosses the Alice slit from left to right at an angle of $\sim 35^{\circ}$ over the neck of the nucleus.

HD 4150 appears in two consecutive 10-minute spectral images with start times of 13:52:04 and 14:02:49 UT, respectively. For the first five minutes of the first exposure, the star is occulted by the comet nucleus. The star remains in the Alice slit for the remainder of the first exposure and the first five minutes of the second exposure. During these exposures, $67 \mathrm{P} / \mathrm{C}-\mathrm{G}$ was $1.30 \mathrm{au}$ from the Sun, Rosetta was orbiting $313 \mathrm{~km}$ from the comet center, and the solar phase angle was $108^{\circ}$.

The spectra of HD 4150 extracted from these exposures are shown in green (first exposure) and brown (second exposure) in Figure 2. The observed stellar fluxes are corrected for the reduced amount of time the star was in the slit during these exposures. Nevertheless, differences between the two exposures are evident; most notably, the first (green) spectrum has less flux from 1350 to $1600 \mathrm{~A}$.

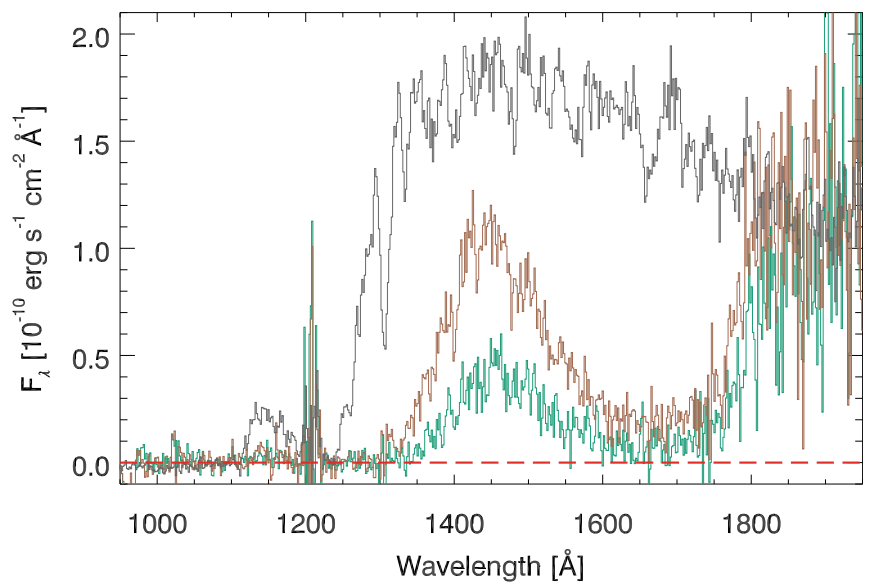

Figure 2. Three spectra of HD 4150 obtained by Alice. The revisit (i.e., intrinsic) spectrum taken on 2016 June 6 is shown in gray, and the postoccultation spectra taken on 2015 September 13 at 13:52:04 and 14:02:49 UT are shown in green and brown, respectively.

HD 4150 was re-observed (revisited) on 2016 June 6 when it was far from the nucleus, at an off-nadir angle of $79^{\circ}$. The purpose of this 15 minute integration, which started at 06:02:24 UT, was to characterize the intrinsic stellar spectrum without the presence of foreground coma absorption (see Keeney et al. 2017 for details). When HD 4150 was revisited, the comet was $3.15 \mathrm{au}$ from the Sun and the solar phase angle was $67^{\circ}$. The intrinsic stellar spectrum is shown in gray in Figure 2, and has considerably more flux from 1250 to $1800 \AA$ than the spectra obtained immediately after the occultation.

The analysis below, and that of Keeney et al. (2017), assumes that the stellar spectrum obtained when the star was revisited while far from the nucleus is equivalent to the intrinsic stellar spectrum (i.e., that there is no foreground coma absorption at that time). Although it is true that $\mathrm{O}_{2}$ is extremely volatile, with a sublimation temperature in vacuum of $\sim 30 \mathrm{~K}$ (Fray \& Schmitt 2009), it has been found to be strongly correlated with $\mathrm{H}_{2} \mathrm{O}$ in the coma of $67 \mathrm{P} / \mathrm{C}-\mathrm{G}$ (Bieler et al. 2015; Fougere et al. 2016), and the production rate of $\mathrm{H}_{2} \mathrm{O}$ was down by two to three orders of magnitude at 3.15 au compared to $1.30 \mathrm{au}$ (Hansen et al. 2016; Gasc et al. 2017). However, even if our assumptions are incorrect and a small amount of foreground coma absorption is present in the revisit spectrum, then this would cause us to underestimate the amount of foreground coma absorption at the time of the occultation. ${ }^{11}$

\section{Results and Analysis}

The spectra of HD 4150 taken immediately after the occultation by $67 \mathrm{P} / \mathrm{C}-\mathrm{G}$ were analyzed analagously to the stellar appulse spectra of Keeney et al. (2017), except in one regard. The ultraviolet (UV)-bright stars that Keeney et al. (2017) used to study the near-nucleus coma all had measurable far-UV flux down to $\sim 900 \AA$ (see Figure 1 of Keeney et al. 2017), but HD 4150 is an A0 star with almost no flux below $\sim 1250 \AA$ (see Figure 2). This lack of flux blueward of $1250 \AA$ has important consequences for our analysis.

Although $\mathrm{H}_{2} \mathrm{O}$ and $\mathrm{O}_{2}$ have relatively large cross sections from 1250 to $1800 \AA$ (Chung et al. 2001; Yoshino et al. 2005), other abundant coma species do not (i.e., $\mathrm{CO}$ and $\mathrm{CO}_{2}$; Cairns \& Samson 1965; Yoshino et al. 1996), which means that they

11 The same can be said for the stellar appulse analysis of Keeney et al. (2017). 
cannot be directly constrained by our data. Fortunately, at the time of the occultation VIRTIS was acquiring data while pointed $\sim 3.6 \mathrm{~km}$ above the sunward limb of the nucleus. Thus, we adopt their contemporaneous column density ratio of $N_{\mathrm{CO}_{2}} / N_{\mathrm{H}_{2} \mathrm{O}}=0.310 \pm 0.034$ (Bockelée-Morvan et al. 2016) for our analysis. The position of the VIRTIS-H aperture with respect to the Alice slit and the path of HD 4150 are shown in Figure 1.

To isolate the coma signature from the intrinsic stellar flux and interstellar absorption, we divided the stellar spectra taken immediately post-occultation (i.e., the green and brown spectra in Figure 2) by the spectrum of the star taken much later (i.e., the gray spectrum in Figure 2), after first scaling them to have the same median flux from 1850 to $1950 \AA$. This procedure reduces our sensitivity to the uncertainty in the amount of time the star was in the slit during our long exposures (Keeney et al. 2017). The resulting normalized spectrum quantifies the amount of foreground coma absorption in the post-occultation exposures.

Keeney et al. (2017) modeled far-UV absorption from 10 molecular species (see their Table 3 and Figure 2 for adopted cross sections) in Alice spectra normalized as above. We use the same procedure here, except that we only fit wavelengths in the range of 1250-1950 $\AA$ because HD 4150 has insufficient flux blueward of $1250 \AA$ (see Figure 2). Consequently, we remove $\mathrm{CO}$ from our fits because it has no appreciable cross section redward of $1050 \AA$ (Cairns \& Samson 1965).

The column density of $\mathrm{H}_{2} \mathrm{O}$ is fit directly and allowed to vary in the range of $\log N_{\mathrm{H}_{2} \mathrm{O}}=14-18$. The abundances of all other species are fit relative to $\mathrm{N}_{\mathrm{H}_{2} \mathrm{O}}, \mathrm{O}_{2}$ is allowed to vary in the range of $N_{\mathrm{O}_{2}} / N_{\mathrm{H}_{2} \mathrm{O}}=0-1, \mathrm{CO}_{2}$ is fixed at the ratio measured by VIRTIS $\left(N_{\mathrm{CO}_{2}} / N_{\mathrm{H}_{2} \mathrm{O}}=0.310 \pm 0.034\right.$; Bockelée-Morvan et al. 2016), and all other species $\left(\mathrm{CH}_{4}, \mathrm{C}_{2} \mathrm{H}_{2}, \mathrm{C}_{2} \mathrm{H}_{6}, \mathrm{C}_{2} \mathrm{H}_{4}\right.$, $\mathrm{C}_{4} \mathrm{H}_{2}$, and $\mathrm{H}_{2} \mathrm{CO}$ ) are constrained to have $N / N_{\mathrm{H}_{2} \mathrm{O}}<0.01$ (Le Roy et al. 2015). All species except $\mathrm{CO}$ and $\mathrm{CO}_{2}$ are treated the same as they were in Keeney et al. (2017). The absorption profiles are determined directly from high-resolution cross sections (1-2 $\AA$ for $\mathrm{H}_{2} \mathrm{O}$ and $\mathrm{O}_{2}$; references for all adopted cross sections are listed in Table 3 of Keeney et al. 2017), then convolved to the spectral resolution of Alice ( $9 \AA$ FWHM for the narrow part of the slit) before being compared to the data.

Our fits to the spectra of HD 4150 taken immediately after its occultation by $67 \mathrm{P} / \mathrm{C}-\mathrm{G}$ are shown in Figures $3-4$. The top panels show the normalized spectra in black, and our best-fit absorption profiles from $\mathrm{H}_{2} \mathrm{O}, \mathrm{O}_{2}, \mathrm{CO}_{2}$, and all other species are shown in blue, green, brown, and purple, respectively. The ensemble fit from all species is shown in pink, and the shaded regions represent $95 \%(2 \sigma)$ confidence bands. Recall that $N_{\mathrm{CO}_{2}} / N_{\mathrm{H}_{2} \mathrm{O}}$ is held fixed at the value measured contemporaneously by VIRTIS (Bockelée-Morvan et al. 2016). The bottom panel shows the residual of the ensemble fit as a function of wavelength, with the $1 \sigma$ flux uncertainty overlaid in orange. Masked regions that are not used to constrain the fits are shown in lighter hues in both panels.

We searched for systematic offsets in the best-fit $\mathrm{H}_{2} \mathrm{O}$ and $\mathrm{O}_{2}$ column densities using Monte Carlo simulations to compare the values retrieved from forward-modeled data with Poissonian noise and known input values. However, unlike in Keeney et al. (2017), we found no evidence for systematic offsets. Reassuringly, the large optical depth of the $\mathrm{H}_{2} \mathrm{O}$ absorption makes the fitting results more robust. Thus, we adopt the $\mathrm{H}_{2} \mathrm{O}$ and $\mathrm{O}_{2}$ column densities returned by our fits as final.

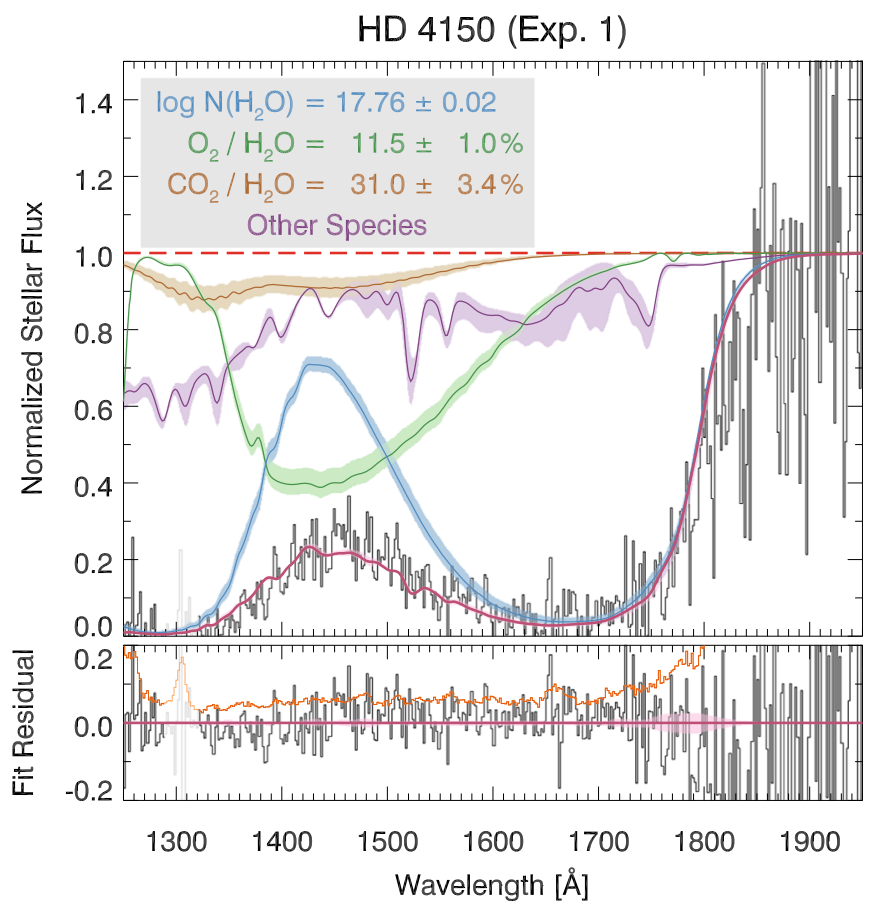

Figure 3. Best-fit column densities for the first post-occultation exposure of HD 4150 , with $95 \%(2 \sigma)$ confidence bands. Top: the normalized stellar flux with the ensemble fit (pink) and individual-species absorption overlaid Bottom: the residual of the ensemble fit with the $1 \sigma$ flux uncertainty (orange) overlaid. Masked regions are shown in lighter hues in both panels; these regions are not used to constrain the fits.

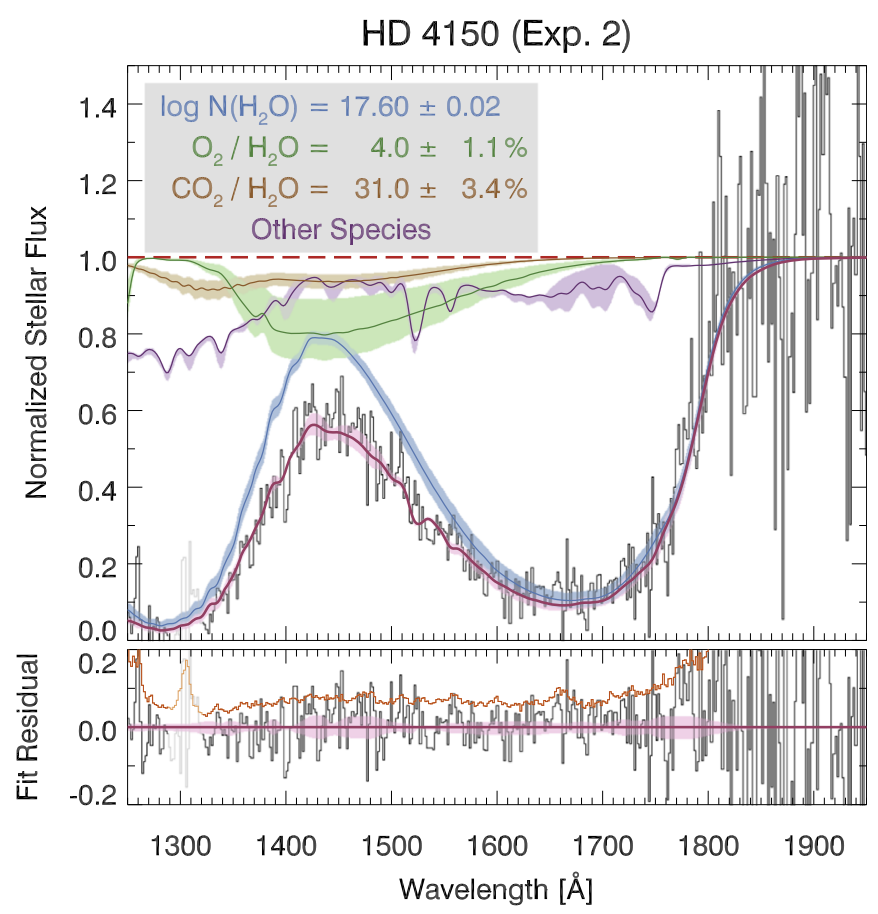

Figure 4. Best-fit column densities for the second post-occultation exposure of HD 4150, with $95 \%(2 \sigma)$ confidence bands.

\section{Discussion}

The decrease in the $\mathrm{H}_{2} \mathrm{O}$ column density from the first exposure of HD 4150 to the second (see Figures 3-4) agrees with the naïve expectation that $N \propto \rho^{-1}$ (Haser 1957), where $\rho$ is the impact parameter with respect to the nucleus. $N_{\mathrm{H}_{2} \mathrm{O}}$ 
decreases by a factor of $\sim 1.4$ between the two exposures when $\rho$ increases by a factor of $\sim 1.3$ (we estimate $\rho \approx 0.3 \mathrm{~km}$ and $0.4 \mathrm{~km}$ for the first and second exposures, respectively; see Figure 1). However, the contemporaneous VIRTIS measurement $\left(\log N_{\mathrm{H}_{2} \mathrm{O}}=17.00 \pm 0.04\right.$ at $\rho \approx 2.7 \mathrm{~km}$; BockeléeMorvan et al. 2016) ${ }^{12}$ is not as consistent with $N \propto \rho^{-1}$, finding a factor of $\sim 6$ decrease in $N_{\mathrm{H}_{2} \mathrm{O}}$ compared to the first post-occultation exposure when $\rho$ increases by a factor of $\sim 9$.

Of course, the Haser (1957) model, which assumes spherically symmetric outflow from a point source, is far too simplistic to be directly applied to data from Rosetta 67P/C-G. From Rosetta's vantage point embedded in the coma of $67 \mathrm{P} / \mathrm{C}$ $\mathrm{G}$, the nucleus is clearly resolved and far from spherical (Figure 1), and observed gas (e.g., Migliorini et al. 2016) and dust (e.g., Gerig et al. 2018) outbursts are not axisymmetric. Thus, it is questionable whether the analytic prediction of Haser (1957; namely, $N \propto \rho^{-1}$ ) holds for the Alice data.

The best-fit values of the $\mathrm{O}_{2}$ column density are $\log N_{\mathrm{O}_{2}}=16.82 \pm 0.04$ for the first post-occultation exposure (Figure 3) and $\log N_{\mathrm{O}_{2}}=16.20 \pm 0.12$ for the second exposure (Figure 4), corresponding to a factor of $\sim 4$ decrease. It is unclear why $N_{\mathrm{O}_{2}}$ is decreasing almost three times more quickly than $\mathrm{N}_{\mathrm{H}_{2} \mathrm{O}}$. Keeney et al. (2017) found no clear trend in $N_{\mathrm{O}_{2}}$ as a function of the impact parameter when $\rho \approx 5-15 \mathrm{~km}$ (see their Figure 9). However, the exposures of HD 4150 probe the coma much closer to the nucleus than any of our previous sight lines, so we cannot confidently extrapolate the Keeney et al. (2017) measurements to $\rho<1 \mathrm{~km}$.

The most puzzling result of Keeney et al. (2017) was that the relative abundance of $\mathrm{O}_{2}$ with respect to $\mathrm{H}_{2} \mathrm{O}$ inferred from the Alice data (median $N_{\mathrm{O}_{2}} / N_{\mathrm{H}_{2} \mathrm{O}}=25 \%$ ) was nearly an order of magnitude larger than the average value of $n_{\mathrm{O}_{2}} / n_{\mathrm{H}_{2} \mathrm{O}}=3.85 \pm 0.85 \%$ found by ROSINA (Bieler et al. 2015). In fact, the data sets almost seemed to be mutually exclusive; Keeney et al. (2017) did not find a single highquality example with $N_{\mathrm{O}_{2}} / N_{\mathrm{H}_{2} \mathrm{O}}<10 \%$ (although there were several upper limits), and ROSINA almost never measured $n_{\mathrm{O}_{2}} / n_{\mathrm{H}_{2} \mathrm{O}}>10 \%$ (Bieler et al. 2015; Fougere et al. 2016). Keeney et al. (2017) speculated that the discrepancy could be attributed to comparing column density along a line of sight near the nucleus with number density measured in situ at the spacecraft position, but no firm conclusions could be drawn. It is therefore reassuring that we measure a relative abundance of $\mathrm{O}_{2} / \mathrm{H}_{2} \mathrm{O}$ that is nearly identical to the average ROSINA value in one of the post-occultation exposures (see Figure 4).

However, whereas Keeney et al. (2017) did not find any instances of $\mathrm{O}_{2} / \mathrm{H}_{2} \mathrm{O}$ consistent with ROSINA measurements in absorption, the emission-line technique of Feldman et al. (2016) frequently infers $\mathrm{O}_{2} / \mathrm{H}_{2} \mathrm{O}$ values near the sunward limb of the nucleus in the Alice data that are consistent with ROSINA values. For example, an estimate of $\mathrm{O}_{2} / \mathrm{H}_{2} \mathrm{O}$ can be derived from exposures taken just before and just after the occultation using the ratio of the semi-forbidden O I] $1356 \AA$ line to $\mathrm{HI} \mathrm{Ly} \beta$. If we assume that all of the OI] $1356 \AA$ emission near the limb comes from electron impact on $\mathrm{H}_{2} \mathrm{O}$ (Makarov et al. 2004), $\mathrm{O}_{2}$ (Kanik et al. 2003), and $\mathrm{CO}_{2}$ (Mumma et al. 1972) at an energy of $100 \mathrm{eV}$, then the brightness of the C I $1657 \AA$ line suggests that $25 \%-30 \%$ of the

\footnotetext{
12 Bockelée-Morvan et al. (2016) list $\rho=3.6 \mathrm{~km}$ for the contemporaneous exposure, which is measured from the center of the nucleus. The value we list is measured with respect to the limb of the nucleus for consistency with Figure 1.
}

$1356 \AA$ brightness comes from electron impact dissociation of $\mathrm{CO}_{2}$. Similarly, large off-nadir steps along the slit suggest that $25 \%-30 \%$ of the HI Ly $\beta$ brightness comes from resonant scattering in the coma. After these corrections, we estimate that $\mathrm{O}_{2} / \mathrm{H}_{2} \mathrm{O} \approx 4 \%$ in the Alice exposures surrounding the occultation. Although this estimate is predicated on many assumptions, it is nevertheless reassuring that it is identical to the value we derived for our second exposure (see Figure 4).

Further, the above estimate is consistent with $\mathrm{O}_{2} / \mathrm{H}_{2} \mathrm{O} \approx 4 \%$ being the quiescent value at this time, whereas the elevated value of $\mathrm{O}_{2} / \mathrm{H}_{2} \mathrm{O} \approx 10 \%$ (Figure 3 ) may be associated with a strong, collimated dust outburst observed in the sunward direction by VIRTIS-H (Bockelée-Morvan et al. 2017) and VIRTIS-M (Rinaldi et al. 2018). The dust outburst peaked at approximately 13:30:00 UT (Rinaldi et al. 2018), 20 minutes before our first post-occultation exposure began. At the beginning of the first Alice exposure, the radiance of the dust emission had decayed to $\sim 20 \%$ of its peak compared to the quiescent level, and by the beginning of the second Alice exposure it had returned to the quiescent level altogether (see Figure 4 of Rinaldi et al. 2018). Thus, one plausible explanation for the differing $\mathrm{O}_{2} / \mathrm{H}_{2} \mathrm{O}$ levels in the two Alice exposures is a non-constant production rate of $\mathrm{O}_{2}$ (i.e., the amount of $\mathrm{O}_{2}$ in the first exposure is affected by the dust outburst). However, Bockelée-Morvan et al. (2017) found no increase in $\mathrm{H}_{2} \mathrm{O}$ or $\mathrm{CO}_{2}$ column density during the outburst, so it is unclear why the $\mathrm{O}_{2}$ production rate would be affected but not those of $\mathrm{H}_{2} \mathrm{O}$ and $\mathrm{CO}_{2}$.

Figure 5 shows $N_{\mathrm{O}_{2}}$ and $N_{\mathrm{O}_{2}} / N_{\mathrm{H}_{2} \mathrm{O}}$ as a function of $N_{\mathrm{H}_{2} \mathrm{O}}$ for the two post-occultation exposures of HD 4150 and the stellar appulses of Keeney et al. (2017). We confirm that $N_{\mathrm{O}_{2}}$ and $N_{\mathrm{H}_{2} \mathrm{O}}$ are strongly correlated as expected due to the strong correlation between $n_{\mathrm{O}_{2}}$ and $n_{\mathrm{H}_{2} \mathrm{O}}$ in the ROSINA data (Bieler et al. 2015; Fougere et al. 2016). Owing to the smaller impact parameters probed, our occultation data find larger $N_{\mathrm{H}_{2} \mathrm{O}}$ values and smaller $N_{\mathrm{O}_{2}} / N_{\mathrm{H}_{2} \mathrm{O}}$ than the appulses, and overall there is a clear trend of decreasing $\mathrm{O}_{2} / \mathrm{H}_{2} \mathrm{O}$ with increasing $N_{\mathrm{H}_{2} \mathrm{O}}$ in the Alice data (e.g., when $N_{\mathrm{H}_{2} \mathrm{O}}<10^{16} \mathrm{~cm}^{-2}$ the median $N_{\mathrm{O}_{2}} / N_{\mathrm{H}_{2} \mathrm{O}}=41 \%$, whereas the median $N_{\mathrm{O}_{2}} / N_{\mathrm{H}_{2} \mathrm{O}}=16 \%$ for larger $N_{\mathrm{H}_{2} \mathrm{O}}$ ).

This trend, which was first noted by Bieler et al. (2015), suggests that we are able to detect an $\mathrm{O}_{2} / \mathrm{H}_{2} \mathrm{O}$ abundance consistent with the ROSINA measurements in Figure 4 simply because the $\mathrm{H}_{2} \mathrm{O}$ column density is sufficiently large. A signalto-noise ratio $(\mathrm{S} / \mathrm{N})$-dependent detection threshold for $N_{\mathrm{O}_{2}}$ in the Alice data is consistent with this trend, but we see no clear evidence that our measurements are strongly affected by such a selection bias (Figure 5). Further, this hypothesis cannot explain all of the Alice measurements since Keeney et al. (2017) found two examples where $N_{\mathrm{O}_{2}} / N_{\mathrm{H}_{2} \mathrm{O}}>40 \%$ when $N_{\mathrm{H}_{2} \mathrm{O}}>10^{16} \mathrm{~cm}^{-2}$. Thus, a full explanation for the discrepancy in the $\mathrm{O}_{2} / \mathrm{H}_{2} \mathrm{O}$ abundance between the Alice and ROSINA measurements remains elusive.

\subsection{Empirical Coma Model Comparisons}

Hansen et al. (2016) developed an empirical coma model to study the evolution of the $\mathrm{H}_{2} \mathrm{O}$ production rate between 2014 June and 2016 May. This model is based on comparisons between the ROSINA data and the direct simulation Monte Carlo models of the 3D neutral gas coma, and corroborated by comparisons with other Rosetta instruments (VIRTIS, MIRO, and RPC) and ground-based dust measurements. Here we compare the predictions of the Hansen et al. (2016) model with 

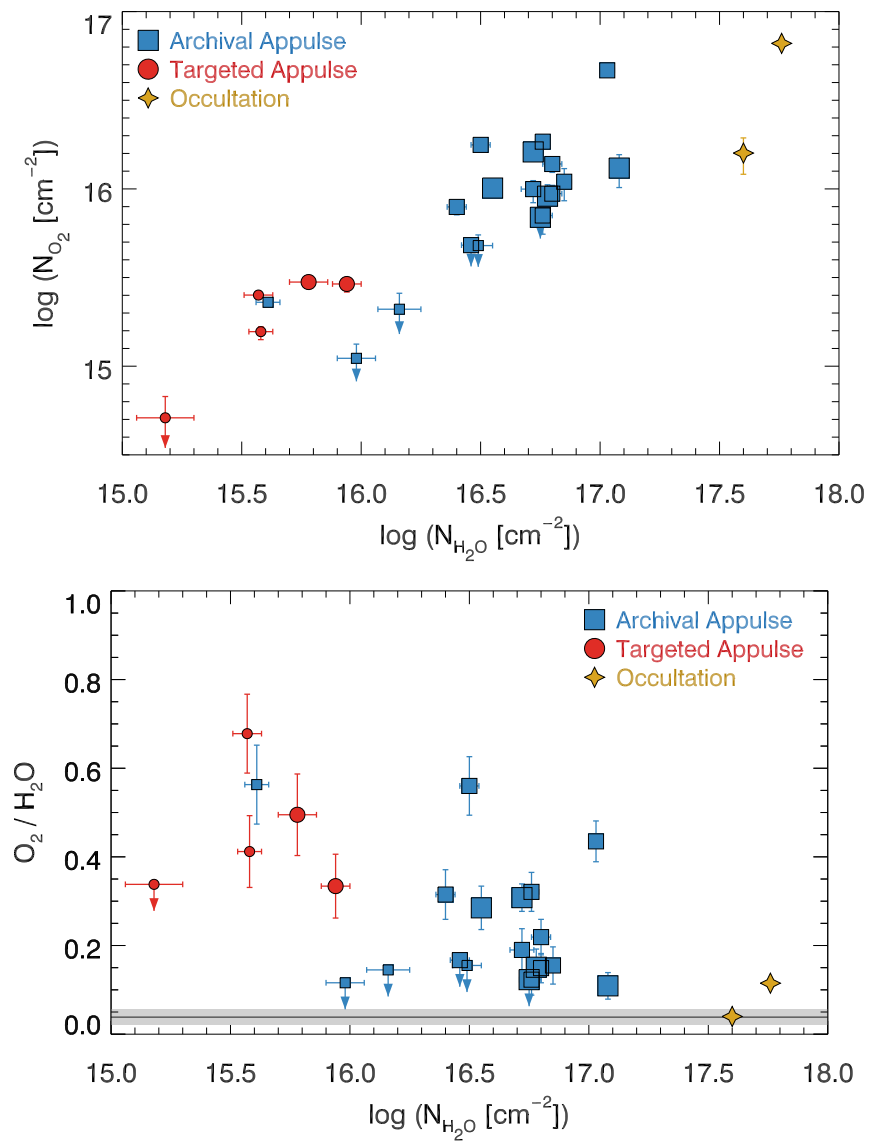

Figure 5. $\mathrm{O}_{2}$ column density (top) and relative abundance of $\mathrm{O}_{2} / \mathrm{H}_{2} \mathrm{O}$ (bottom) as a function of $N_{\mathrm{H}_{2} \mathrm{O}}$ for the two post-occultation exposures of $\mathrm{HD} 4150$ and the stellar appulses of Keeney et al. (2017). There are clear trends of increasing $N_{\mathrm{O}_{2}}$ and decreasing $\mathrm{O}_{2} / \mathrm{H}_{2} \mathrm{O}$ with increasing $N_{\mathrm{H}_{2} \mathrm{O}}$. The shaded area in the bottom panel is the $95 \%(2 \sigma)$ confidence band for $n_{\mathrm{O}_{2}} / n_{\mathrm{H}_{2} \mathrm{O}}$ from Bieler et al. (2015).

$\mathrm{H}_{2} \mathrm{O}$ column densities measured by Alice from stellar appulses and occultations.

Hansen et al. (2016) parameterize the number density, $n$, of $\mathrm{H}_{2} \mathrm{O}$ molecules as

$$
n=\frac{f Q}{4 \pi r^{2} v}
$$

where $Q$ is the $\mathrm{H}_{2} \mathrm{O}$ production rate, $r$ is the distance from the comet center, $v$ is the gas velocity, and $f$ is an empirical correction factor. When $f=1$, Equation (1) is equivalent to spherically symmetric radial expansion, otherwise it accounts for the observed anisotropy in the coma of 67P/C-G (Fougere et al. 2016; Hansen et al. 2016; Läuter et al. 2019). Between the equinoxes (2015 May to 2016 March) the factors $Q, v$, and $f$ are independent of $r$ (see Tables 1 and 2 of Hansen et al. 2016), so the number density can be integrated to find the column density,

$$
\begin{aligned}
N & =\frac{f Q}{4 \pi v} \int_{0}^{\infty} \frac{d s}{r^{2}(s)} \\
& =\frac{f Q}{4 \pi v} \int_{0}^{\infty} \frac{d s}{s^{2}+r_{\mathrm{sc}}^{2}-2 s r_{\mathrm{sc}} \cos \theta}
\end{aligned}
$$

where $r_{\mathrm{sc}}$ is the distance from the spacecraft to the comet center, $\theta$ is the angle of the sight line with respect to the comet

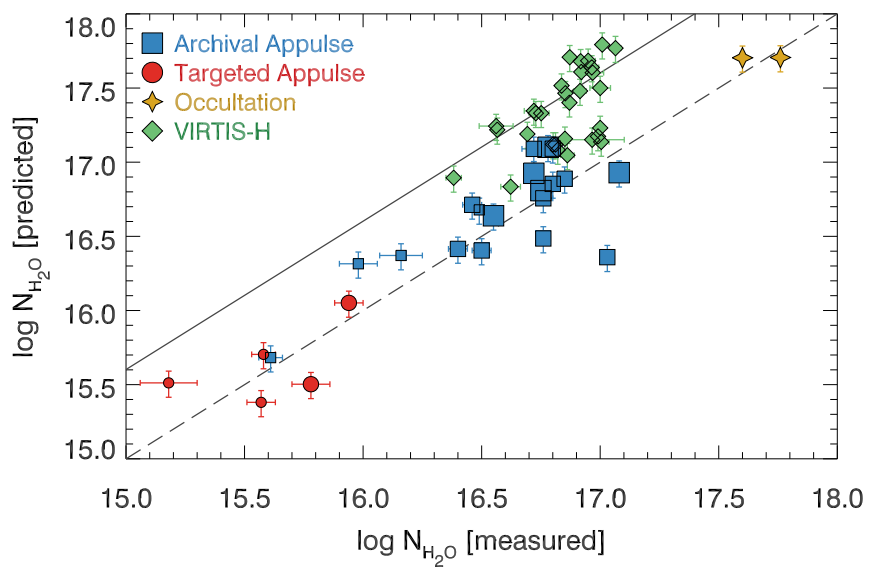

Figure 6. Predicted $\mathrm{H}_{2} \mathrm{O}$ column density from Equation (2) compared to the Alice and VIRTIS measurements. The dashed line shows perfect agreement between the predicted and measured values, and the dotted-dashed line shows the predicted values that are four times larger than measured.

center, and $r^{2}(s)$ is given by the law of cosines. The integration is performed along the line of sight, $s$, starting from the spacecraft position $(s \equiv 0)$.

Prior to the inbound equinox, $f$ is a function of both $R_{h}$ and $r$ (Hansen et al. 2016) and cannot be separated from the integral. $Q$ and $v$ are defined in Equations (7) and (10) of Hansen et al. (2016), and depend only on the heliocentric distance,

$$
\begin{aligned}
& Q\left(R_{h}\right)= \begin{cases}(2.58 \pm 0.12) R_{h}^{-5.10 \pm 0.05}, & \text { pre-perihelion } \\
(15.8 \pm 0.9) R_{h}^{-7.15 \pm 0.08}, & \text { post-perihelion }\end{cases} \\
& v\left(R_{h}\right)=\left(771.0-55.5 R_{h}\right)\left(1+0.171 e^{-\frac{R_{h}-1.24}{0.13}}\right),
\end{aligned}
$$

where $Q$ has units of $10^{28}$ molecules $\mathrm{s}^{-1}$ and $v$ has units of $\mathrm{m} \mathrm{s}^{-1}$.

Figure 6 shows the predicted $\mathrm{H}_{2} \mathrm{O}$ column density from Equation (2) compared to the Alice and VIRTIS measurements near perihelion. The uncertainty on the model predictions is assumed to be $20 \%$ (Hansen et al. 2016). The Alice data are consistent with the predicted values over the full range of column densities measured. Using all data obtained between the equinoxes (all but one data point; see Figure 7), where the assumptions behind Equation (2) are valid, the rms difference between the Alice measurements and the Hansen et al. (2016) prediction is 0.24 dex.

However, for VIRTIS data (Bockelée-Morvan et al. 2016), the predicted column densities are systematically higher than the measurements. Fougere et al. (2016) had to reduce the $\mathrm{H}_{2} \mathrm{O}$ column densities predicted by their model by a factor of four to match the measurements of Bockelée-Morvan et al. (2016). The dotted-dashed line in Figure 6 indicates a model overprediction by a factor of four and passes through much of the VIRTIS data, agreeing with the analysis of Fougere et al. (2016).

As a final check on the consistency of the Alice data and the Hansen et al. (2016) coma model, Equation (2) can be rearranged so that the details of the observing geometry cancel out, leaving only a predicted trend with heliocentric distance,

$$
\frac{Q\left(R_{h}\right)}{v\left(R_{h}\right)}=\frac{4 \pi N / f}{\int_{0}^{\infty}\left(s^{2}+r_{\mathrm{sc}}^{2}-2 s r_{\mathrm{sc}} \cos \theta\right)^{-1} d s} .
$$




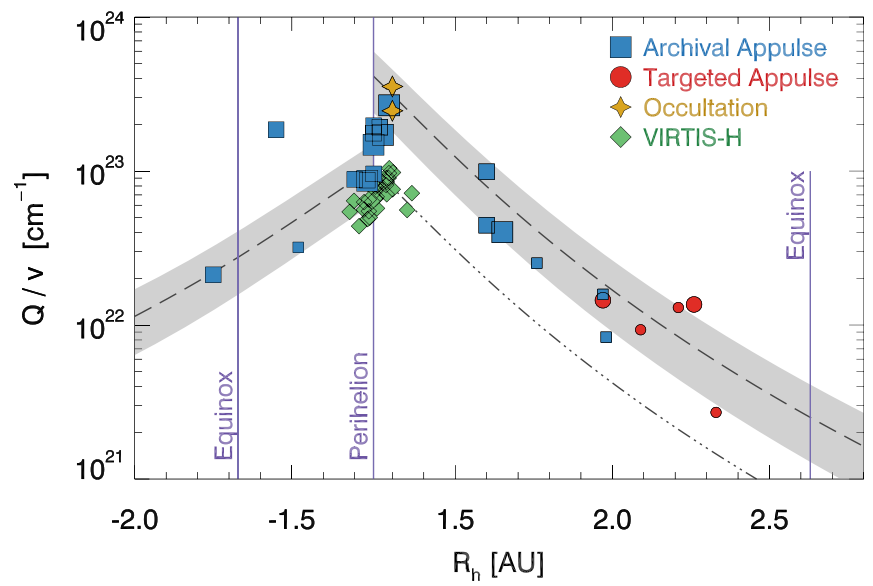

Figure 7. Predicted trend of $Q / v$ as a function of $R_{h}$ (Hansen et al. 2016) compared to values inferred from the Alice and VIRTIS measurements using Equation (3). The shaded region shows the $95 \%(2 \sigma)$ confidence band for $Q / v$ and the dashed lines show the best-fit relation from Hansen et al. (2016); the dotted-dashed line post-perihelion shows the Hansen et al. (2016) prediction reduced by a factor of four.

Figure 7 shows the Hansen et al. (2016) prediction of $Q / v$ as a function of $R_{h}$ compared to values derived from the Alice and VIRTIS measurements using Equation (3). The dashed lines show the best-fit relation of Hansen et al. (2016), and the gray shaded regions show the $95 \%(2 \sigma)$ confidence band for the prediction. The Alice appulse (Keeney et al. 2017) and occultation measurements (Figures 3-4) are consistent with the Hansen et al. (2016) prediction, except very close to perihelion where the Hansen et al. (2016) model is discontinuous.

The VIRTIS-H data are lower than the Hansen et al. (2016) prediction over the full range of heliocentric distance, and show no discontinuity at perihelion. The disagreement is largest after perihelion, where the VIRTIS data are lower than the prediction by a factor of $\sim 4$ (dotted-dashed line). Fougere et al. (2016) discussed possible reasons for this discrepancy and we will not speculate further, except to note that it is reassuring that we are able to reproduce the reported tension.

Furthermore, the fact that our $\mathrm{H}_{2} \mathrm{O}$ measurements are consistent with the predictions of the Hansen et al. (2016) coma model increases confidence in our ability to directly compare results from Alice and ROSINA. On the other hand, the broad consistency in $\mathrm{H}_{2} \mathrm{O}$ results between the two instruments makes the discrepancy in $\mathrm{O}_{2} / \mathrm{H}_{2} \mathrm{O}$ values all the more puzzling.

\subsection{Modeling Uncertainties}

There are several sources of systematic uncertainty in our modeling process, as detailed in Keeney et al. (2017). Perhaps the hardest to quantify is the uncertainty introduced by unknown far-UV absorption cross sections. ROSINA-DFMS has found dozens of species in the coma of 67P/C-G (Le Roy et al. 2015; Altwegg et al. 2017), many of which have no measured far-UV cross sections, and could thus conceivably have a large enough cross section that even a trace amount could produce appreciable absorption. We believe this circumstance is unlikely but cannot rule it out.

Another concern is the temperature dependence of the adopted cross sections, because all of the cross sections we use were measured at $250-300 \mathrm{~K}$ (see Table 3 of Keeney et al. 2017), and the coma is expected to cool adiabatically. There are several reasons for this choice. First, high-resolution laboratory measurements are not consistently available for all of the modeled species at any other temperature. Adopting a consistent temperature for the cross sections of all of the modeled species minimizes the uncertainty introduced by cross sections for different species having different behavior with decreasing temperature.

Reassuringly, Figure 6 shows that the Alice measurements of $\mathrm{H}_{2} \mathrm{O}$, derived from absorption cross sections measured at $250 \mathrm{~K}$ (Chung et al. 2001), are consistent with the predictions of the Hansen et al. (2016) empirical coma model for 67P/C-G. Thus, if temperature-dependent cross sections are invoked to explain different $\mathrm{O}_{2} / \mathrm{H}_{2} \mathrm{O}$ measurements from ROSINA and Alice, they must preferentially affect $\mathrm{O}_{2}$ or else the Alice and ROSINA $\mathrm{H}_{2} \mathrm{O}$ values would disagree.

Finally, and most speculatively, there are $\mathrm{O}_{2}$ and $\mathrm{H}_{2} \mathrm{O}$ crosssection measurements at multiple temperatures over part of the modeled far-UV wavelength range which suggest that using room temperature cross sections yields lower $\mathrm{O}_{2} / \mathrm{H}_{2} \mathrm{O}$ values than at lower temperatures. Low-resolution measurements (Yoshino et al. 2005) indicate that the peak $\mathrm{O}_{2}$ cross section at $\sim 1400 \AA$ decreases by $\sim 0.1 \mathrm{dex}$ as the temperature decreases from 295 to $78 \mathrm{~K}$, implying that a larger $\mathrm{O}_{2}$ column density is required to match the observed absorption at lower temperatures. High-resolution $\mathrm{H}_{2} \mathrm{O}$ cross sections are available at $250 \mathrm{~K}$ (Chung et al. 2001) and $298 \mathrm{~K}$ (Mota et al. 2005), and the peak cross section at $\sim 1650 \AA$ is $\sim 0.1$ dex larger at $250 \mathrm{~K}$, implying that a smaller $\mathrm{H}_{2} \mathrm{O}$ column density is required to match the observed absorption at lower temperatures. Although we cannot be confident that the $\mathrm{H}_{2} \mathrm{O}$ cross section continues to increase at temperatures $<250 \mathrm{~K}$, the existing data indicate that lower temperatures necessitate larger $\mathrm{O}_{2} / \mathrm{H}_{2} \mathrm{O}$ to fit the data, further suggesting that adopting room temperature cross sections is not the cause of the discrepancy between our $\mathrm{O}_{2} / \mathrm{H}_{2} \mathrm{O}$ values and the ROSINA measurements.

\section{Summary}

We have presented far-UV spectra of the A0 IV star HD 4150, which was serendipitously observed by Rosetta's Alice imaging spectrometer on 2015 September 13. HD 4150 was observed in two 10-minute integrations immediately after being occulted by $67 \mathrm{P} / \mathrm{C}-\mathrm{G}$, and revisited approximately nine months later when its line of sight was far from the nucleus. By comparing the two epochs of stellar spectra, we were able to quantify the amount of $\mathrm{H}_{2} \mathrm{O}$ and $\mathrm{O}_{2}$ within $1 \mathrm{~km}$ of the nucleus.

We find that $N_{\mathrm{H}_{2} \mathrm{O}} \propto \rho^{-1}$ in our consecutive exposures of HD 4150 , but $N_{\mathrm{O}_{2}}$ decreases $\sim 3$ times faster than $N_{\mathrm{H}_{2} \mathrm{O}}$. We have also measured a value of $N_{\mathrm{O}_{2}} / N_{\mathrm{H}_{2} \mathrm{O}}$ that is consistent with ROSINA measurements of $n_{\mathrm{O}_{2}} / n_{\mathrm{H}_{2} \mathrm{O}}$ (Bieler et al. 2015; Fougere et al. 2016). Combining our observations of HD 4150 with previous results from Keeney et al. (2017), we confirm the strong correlation between $\mathrm{O}_{2}$ and $\mathrm{H}_{2} \mathrm{O}$ (see top panel of Figure 5, which demonstrates that $N_{\mathrm{O}_{2}}$ increases as $N_{\mathrm{H}_{2} \mathrm{O}}$ increases) first reported by ROSINA (Bieler et al. 2015; Fougere et al. 2016), but find a general decrease in $\mathrm{O}_{2} / \mathrm{H}_{2} \mathrm{O}$ with increasing $N_{\mathrm{H}_{2} \mathrm{O}}$ in the Alice data (see bottom panel of Figure 5), even though the HD 4150 data in isolation suggest otherwise. This trend of decreasing $\mathrm{O}_{2} / \mathrm{H}_{2} \mathrm{O}$ with increasing 
$N_{\mathrm{H}_{2} \mathrm{O}}$ partially explains the initial discrepancy in $\mathrm{O}_{2} / \mathrm{H}_{2} \mathrm{O}$ between Alice and ROSINA.

Several Rosetta instruments (e.g., Alice, ROSINA, VIRTIS, and MIRO) can detect $\mathrm{H}_{2} \mathrm{O}$ in the coma of $67 \mathrm{P} / \mathrm{C}-\mathrm{G}$. However, only Alice and ROSINA can directly detect $\mathrm{O}_{2}$, so the differing Alice and ROSINA measurements of $\mathrm{O}_{2} / \mathrm{H}_{2} \mathrm{O}$ in the coma of $67 \mathrm{P} / \mathrm{C}-\mathrm{G}$ remain mysterious, especially since we have shown that our $\mathrm{H}_{2} \mathrm{O}$ measurements are consistent with the empirical coma model of Hansen et al. (2016).

We have investigated several potential sources of this discrepancy, but none have provided a satisfactory explanation. We note, however, that additional high-resolution laboratory measurements of molecular absorption cross sections in the far$\mathrm{UV}$ at temperatures of $\sim 100 \mathrm{~K}$ would be welcome.

One avenue of future study is modeling the $\mathrm{O}_{2} / \mathrm{H}_{2} \mathrm{O}$ distribution throughout the coma of $67 \mathrm{P} / \mathrm{C}-\mathrm{G}$, then integrating along the Alice line of sight to predict the observed column density ratio. The models of Hansen et al. (2016), Fougere et al. (2016), and Läuter et al. (2019) are based off of in situ ROSINA samples of the coma density and composition, and largely agree with the emission-line measurements from MIRO (Biver et al. 2015; Lee et al. 2015), VIRTIS (Bockelée-Morvan et al. 2015; Fink et al. 2016), and Alice (Feldman et al. 2016). However, emission-line intensities are far more sensitive to density than absorption ( $n^{2}$ compared to $n$ dependence), so a distributed source of $\mathrm{O}_{2}$ would preferentially reveal itself in absorption.

Rosetta is an ESA mission with contributions from its member states and NASA. The authors thank the members of the Rosetta Science Ground System and Mission Operations Center teams, in particular Richard Moissl and Michael Küppers, for their expert and dedicated help in planning and executing the Alice observations. The Alice team acknowledges continuing support from NASA via Jet Propulsion Laboratory contract 1336850 to the Southwest Research Institute. This research has made use of the SIMBAD database, operated at CDS, Strasbourg, France.

Facility: Rosetta (Alice).

\section{ORCID iDs}

Brian A. Keeney (ib https://orcid.org/0000-0003-0797-5313
Paul D. Feldman (1D https://orcid.org/0000-0002-9318-259X

Matthew M. Knight (i) https://orcid.org/0000-0003-2781-6897

John Noonan (iD https://orcid.org/0000-0003-2152-6987

Andrew J. Steffl (1) https://orcid.org/0000-0002-5358-392X

Ronald J. Vervack, Jr. (1) https://orcid.org/0000-00028227-9564

Harold A. Weaver (iD https://orcid.org/0000-0003-0951-7762

\section{References}

Altwegg, K., Balsiger, H., Berthelier, J. J., et al. 2017, MNRAS, 469, S130 Balsiger, H., Altwegg, K., Bochsler, P., et al. 2007, SSRv, 128, 745 Bieler, A., Altwegg, K., Balsiger, H., et al. 2015, Natur, 526, 678 Biver, N., Hofstadter, M., Gulkis, S., et al. 2015, A\&A, 583, A3 Bockelée-Morvan, D., Crovisier, J., Erard, S., et al. 2016, MNRAS, 462, S170 Bockelée-Morvan, D., Debout, V., Erard, S., et al. 2015, A\&A, 583, A6 Bockelée-Morvan, D., Rinaldi, G., Erard, S., et al. 2017, MNRAS, 469, S443 Cairns, R. B., \& Samson, J. A. R. 1965, JGR, 70, 99

Chung, C.-Y., Chew, E. P., Cheng, B.-M., Bahou, M., \& Lee, Y.-P. 2001, NIMPA, 467, 1572

Coradini, A., Capaccioni, F., Drossart, P., et al. 2007, SSRv, 128, 529

Feldman, P. D., A'Hearn, M. F., Feaga, L. M., et al. 2016, ApJL, 825, L8

Fink, U., Doose, L., Rinaldi, G., et al. 2016, Icar, 277, 78

Fougere, N., Altwegg, K., Berthelier, J.-J., et al. 2016, MNRAS, 462, S156

Fray, N., \& Schmitt, B. 2009, P\&SS, 57, 2053

Gasc, S., Altwegg, K., Balsiger, H., et al. 2017, MNRAS, 469, S108

Gerig, S.-B., Marschall, R., Thomas, N., et al. 2018, Icar, 311, 1

Gulkis, S., Frerking, M., Crovisier, J., et al. 2007, SSRv, 128, 561

Hansen, K. C., Altwegg, K., Berthelier, J.-J., et al. 2016, MNRAS, 462, S491 Haser, L. 1957, BSRSL, 43, 740

Kanik, I., Noren, C., Makarov, O. P., et al. 2003, JGRE, 108, 5126

Keeney, B. A., Stern, S. A., A'Hearn, M. F., et al. 2017, MNRAS, 469, S158

Läuter, M., Kramer, T., Rubin, M., \& Altwegg, K. 2019, MNRAS, 483, 852

Le Roy, L., Altwegg, K., Balsiger, H., et al. 2015, A\&A, 583, A1

Lee, S., von Allmen, P., Allen, M., et al. 2015, A\&A, 583, A5

Makarov, O. P., Ajello, J. M., Vattipalle, P., et al. 2004, JGRA, 109, A09303

Migliorini, A., Piccioni, G., Capaccioni, F., et al. 2016, A\&A, 589, A45

Mota, R., Parafita, R., Giuliani, A., et al. 2005, CPL, 416, 152

Mumma, M. J., Stone, E. J., Borst, W. L., \& Zipf, E. C. 1972, JChPh, 57, 68

Pineau, J. P., Parker, J. W., Steffl, A. J., et al. 2019, JSpRo, in press

Rinaldi, G., Bockelée-Morvan, D., Ciarniello, M., et al. 2018, MNRAS, 481, 1235

Stern, S. A., Slater, D. C., Scherrer, J., et al. 2007, SSRv, 128, 507

Yoshino, K., Esmond, J. R., Sun, Y., et al. 1996, JQSRT, 55, 53

Yoshino, K., Parkinson, W. H., Ito, K., \& Matsui, T. 2005, JMoSp, 229, 238 